\title{
Comparison of Central Corneal Thickness Measurements by Pentacam and Ultrasound Pachymetry in Normal Myopic Patients
}

\author{
Sherif Emerah ${ }^{1,2}$, Ehab ELZakzouk ${ }^{1}$, Mohamed Farag $^{1}$
}

${ }^{1}$ Associate Professor of Ophthalmology, Ophthalmology department, Research Institute of Ophthalmology, Giza, Egypt

${ }^{2}$ Eye Hospital, Dubai, United Arab Emirates

Type of article: Original

\begin{abstract}
Introduction: Precise measurement of corneal thickness is of crucial importance in corneal refractive procedures. The aim of this work is to compare the central corneal thickness measurements using Scheimpflug imaging (Pentacam) and contact US pachymetry in normal myopic patients.

Methods: This retrospective cohort study was conducted on in 144 eyes of 72 normal myopic patients during 6 months. The study location was the Research Institute of Ophthalmology (Giza, Egypt), and Magrabi Eye Hospital (Dubai, United Arab Emirates). Central corneal thickness was measured with the two devices. Data were analyzed by descriptive statistics, independent-samples t-test, paired-samples t-test, Mann-Whitney $U$ test and Pearson-Product Moment Correlation Coefficient.

Results: The mean age was $25.3 \pm 5.6$ years (range 18 to 38 years). The median age for our patients is 24 years. The average measurements of CCT were $526.8 \pm 35.3$ and $529.1 \pm 37.9$ for the Pentacam pachymetry and US pachymetry values, respectively. Mean difference between both measurements was $2.3 \pm 2.6 \mu \mathrm{m}$ which is statistically insignificant $(\mathrm{p}=0.08)$. Bland Altman Plot shows border line agreement of the two methods US pachymetry and Pentacam in detection of central corneal thickness.

Conclusion: The results of this study showed no significant differences in CCT readings between the measurements obtained by Pentacam and US pachymetry in normal myopic patients.

Keywords: Corneal thickness, Pentacam, Ultrasound pachymetry
\end{abstract}

\section{Introduction}

Precise measurement of corneal thickness is of crucial importance in corneal refractive procedures, especially laser in situ keratomileusis (LASIK), Photorefractive keratectomy (PRK) and LASIK enhancement. This measurement determines the amount of safe stromal ablation possible to ensure satisfactory outcome to avoid post-operative complications. Corneal thickness also gives information regarding corneal endothelial cell function (1). Normal central corneal thickness (CCT) is 490-560 $\mu \mathrm{m}$ and corneal thickness is greatest at limbus (2). CCT measurements play a significant role in the diagnosis and management of glaucoma, because the value of intraocular pressure should be adjusted according to CCT (3). CCT also plays a very important role in the diagnosis of corneal diseases, such as Fuchs' corneal dystrophy and keratoconus (4). Therefore, evaluating different methods of CCT measurements is of special concern. Many tools have been developed to assess corneal thickness including ultrasound pachymetry, ultrasound biomicroscopy (UBM), the Scheimpflug system, optical biometry, and optical coherence tomography (OCT). The Pentacam HR device (Oculus, Inc.) non-invasive instrument uses a rotating Scheimpflug camera to acquire cross-sectional images of the cornea and lens. Data are calculated from up to 138,000 points. It provides several information details of anterior segment imaging, anterior and posterior corneal topography, and corneal pachymetry. Evaluation of the corneal thickness measurements in its whole area is enabled by the pachymetry map which provides various corneal points such as at geometric center, pupil center and the

\section{Corresponding author:}

Associate Professor Dr. Sherif Emerah, Ophthalmology Department, Research Institute of Ophthalmology, Giza, Egypt. Tel: +201222459388, E-Mail: sherifemera@yahoo.com

Received: June 02, 2016, Accepted: August 14, 2016, Published: December 2016

iThenticate screening: August 14, 2016, English editing: October 10, 2016, Quality control: November 02, 2016

(C) 2016 The Authors. This is an open access article under the terms of the Creative Commons Attribution-NonCommercialNoDerivs License, which permits use and distribution in any medium, provided the original work is properly cited, the use is non-commercial and no modifications or adaptations are made. 
thinnest point. The thickness of the entire cornea can be obtained by Pentacam which determines the front and back surfaces in the corneal tomography. The aim of this work is to compare the central corneal thickness measurements using Scheimpflug imaging (Pentacam) and contact US pachymetry in normal myopic patients.

\section{Material and Methods}

This prospective cohort study was carried out on 144 eyes of 72 patients during 6 months. The study location was the Research Institute of Ophthalmology (Giza, Egypt), and Magrabi Eye Hospital (Dubai, United Arab Emirates). CCT was measured for all eyes using Pentacam (Oculus Inc., Germany), and ultrasound pachymetry (US Pachymetry) (US 4000; Nidek, Japan). Exclusion criteria were patients with corneal diseases such as corneal scarring, opacities and Fuch's endothelial dystrophy or history of previous corneal surgery. Removal of contact lenses was required at least 72 hours before measurement. With Pentacam, the patient's face is accurately aligned to a fixed target within the device. A real time image of the patient's eye is shown to the examiner on the computer screen, with the machine marking the pupil edge and center, and the corneal apex. The image is focused and centered manually. Pentacam's automatic release mode is used in order to reduce operator-dependent variables. Multiple slit images (depending on the settings, was set to 25 or 50) of the anterior segment with 500 true elevation points, are captured by the rotating camera. At the same time, the second camera simultaneously captures and corrects minute eye movements. The entire cornea's single point pachymetric measurements are determined from the calculated front and back corneal surfaces. Only when the "examination quality specification" reading was satisfactory, was it recorded; otherwise it was excluded and remeasured until three valid readings were obtained. For the ultrasound pachymetry, the cornea was anesthetized with topical $0.5 \%$ proparacaine hydrochloride (Alcaine; Alcon Laboratories, TX, USA). The subject was seated on the chair and asked to look straight ahead. The examiner placed the pachymeter probe on the central cornea as perpendicular as possible. Then, five readings were taken. The mean values were used for assessment. Data were analyzed by SPSS version 15 (SPSS Inc., Chicago, Illinois, USA) using descriptive statistics, independent-samples t-test, paired-samples t-test, Mann-Whitney U test and PearsonProduct Moment Correlation Coefficient. A "p-value" of less than 0.05 was considered statistically significant. Bland Altman plot was used to evaluate the agreement among two measurements techniques.

\section{Results}

In this study 144 eyes of 72 subjects were enrolled (28 males and 44 females). The mean age was $25.3 \pm 5.6$ years (range 18 to 38 years). The median age for our patients is 24 years. According to our findings, the average measurements of CCT were $526.8 \pm 35.3$ and $529.1 \pm 37.9$ for the Pentacam pachymetry and US pachymetry values, respectively. Mean difference between both measurements was $2.3 \pm 2.6 \mu \mathrm{m}$.

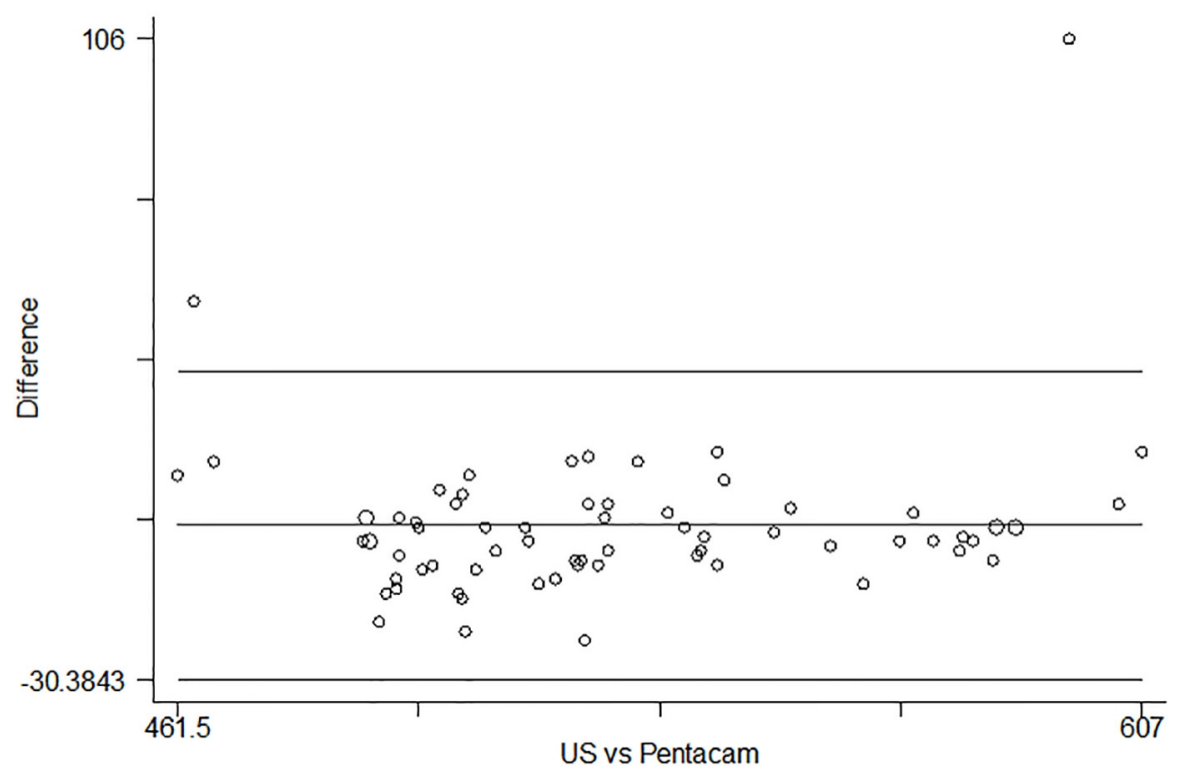

Figure 1. Bland Altman Plot for agreement between US pachymetry and Pentacam in measurement of central corneal thickness. 
Paired-samples t-test showed a lower mean (but no statistically significant difference) of central corneal thickness by Pentacam compared to US pachymetry $(\mathrm{p}=0.08)$. However, the correlational analysis showed a significant positive correlation between central corneal thickness by US Pachymetry and Pentacam $(p=0.000)$. Figure 1 shows border line agreement of the two methods US Pachymetry and Pentacam in detection of central corneal thickness.

\section{Discussion and conclusions}

According to our findings, there were no significant differences in CCT readings between the measurements obtained by two devices (Oculus Pentacam and US pachymetry) in normal myopic patients. Ho et al. demonstrated that the Pentacam significantly underestimates CCT compared with US pachymetry (6). In addition, Hashemi et al. showed that Pentacam underestimates CCT in comparison to US Pachymetry in a study of eyes that had LASIK (7). Some studies evaluated CCT using the Pentacam and US pachymetry in post Lasik eyes; Ciolino et al. demonstrated that CCT measurements performed using the Pentacam have good correlation and agreement with those performed using ultrasonic pachymeter; hence, they concluded that Pentacam can substitute US pachymetry in CCT measurement (8). Similar results had been reported in prospective, cross-sectional study done by Sedaghat et al. The study conducted to evaluate and compare central corneal thickness measurements using rotating Scheimpflug camera, scanning-slit topography, and US pachymetry in virgin, healthy corneas. According to their results, highest agreement was between ultrasonic and Pentacam. They concluded that US pachymetry can be used as a substitute with Pentacam to measuring CCT (9). Similarly, Hani et al. concluded that Pentacam tends to overestimate CCT compared to US pachymetry in post Lasik patients, and he reported that Pentacam probably cannot be used interchangeably with an ultrasound pachymeter (10). Tai et al. had reported that the mean CCT measurements by Pentacam were $10 \mu \mathrm{m}$ more than that obtained using US pachymetry (11). Recently, Sadoughi et al. had compared US pachymetry and Orbscan for the measurement of CCT in normal eyes. They concluded that Orbscan II has good relationship with US pachymetry for measuring CCT in normal eyes however there is a tendency for Orbscan to overestimate CCT as compared to ultrasound pachymetry (12).

\section{Acknowledgments:}

The authors thank the research Institute of Ophthalmology and Magrabi Eye hospital Dubai for supporting this work.

\section{Conflict of Interest:}

There is no conflict of interest to be declared.

\section{Authors' contributions:}

All authors contributed to this project and article equally. All authors read and approved the final manuscript.

\section{References:}

1) Kanski JJ. Clinical Ophthalmology A systematic approach. 5th ed. London: Butterworth-Heineman. 2003: 100 .

2) Cheng H, Bates AK, Wood L, McPherson K. Positive correlation of corneal thickness and endothelial cell loss. Serial measurements after cataract surgery. Arch Ophthalmol. 1988; 106(7): 920-2. doi: 10.1001/archopht.1988.01060140066026. PMID: 3390055.

3) Doughty MJ, Zaman ML. Human corneal thickness and its impact on intraocular pressure measures: a review and metaanalysis approach. Surv Ophthalmol. 2000; 44(5): 367-408. doi: 10.1016/S00396257(00)00110-7. PMID: 10734239.

4) Auffarth GU, Wang L, Völcker HE. Keratoconus evaluation using the Orbscan topography system. Journal of Cataract and Refractive Surgery. 2000; 26(2): 222-8. doi: 10.1016/S0886-3350(99)00355-7.

5) Amano S, Honda N, Amano Y, Yamagami S, Miyai T, Samejima T, et al. Comparison of central corneal thickness measurements by rotating Scheimpflug camera, ultrasonic pachymetry, and scanning-slit corneal topography. Ophthalmology. 2006; 113(6): 937-41. doi: 10.1016/j.ophtha.2006.01.063. PMID: 16751036.

6) Ho T, Cheng AC, Rao SK, Lau S, Leung CK, Lam DS. Central corneal thickness measurements using Orbscan II, Visante, ultrasound, and Pentacam pachymetry after laser in situ keratomileusis for myopia. J Cataract Refract Surg. 2007; 33(7): 1177-82. doi: 10.1016/j.jcrs.2007.03.028. PMID: 17586372.

7) Hashemi H, Mehravaran S. Central corneal thickness measurement with Pentacam, Orbscan II, and ultrasound devices before and after laser refractive surgery for myopia. J Cataract Refract Surg. 2007; 33(10): 1701-7. doi: 10.1016/j.jcrs.2007.05.040. PMID: 17889763. 
8) Ciolino JB, Khachikian SS, Belin MW. Comparison of corneal thickness measurements by ultrasound and Scheimpflug photography in eyes that have undergone laser in situ keratomileusis. Am J Ophthalmol. 2008; 145(1): 75-80. doi: 10.1016/j.ajo.2007.08.026. PMID: 17988643.

9) Sedaghat MR, Daneshvar R, Kargozar A, Derakhshan A, Daraei M. Comparison of central corneal thickness measurement using ultrasonic pachymetry, rotating Scheimpflug camera, and scanning-slit topography. Am J Ophthalmol. 2010; 150(6): 780-9. doi: 10.1016/j.ajo.2010.06.013. PMID: 20932508.

10) Al-Mezaine HS, Al-Amro SA, Kangave D, Al-Obeidan S, Al-Jubair KM. Comparison of central corneal thickness measurements using Pentacam and ultrasonic pachymetry in post-LASIK eyes for myopia. Eur J Ophthal. 2010; 20(5): 852-7. PMID: 20815096.

11) Tai LY, Khaw KW, Ng CM, Subrayan V. Central corneal thickness measurements with different imaging devices and ultrasound pachymetry. Cornea. 2013; 32(6): 766-1. doi: 10.1097/ICO.0b013e318269938d. PMID: 23095499.

12) Sadoughi MM, Einollahi B, Einollahi N, Rezaei J, Roshandel D, Feizi S. Measurement of Central Corneal Thickness Using Ultrasound Pachymetry and Orbscan II in Normal Eyes. J Ophthalmic Vis Res. 2015; 10(1): 4-9. doi: 10.4103/2008-322X.156084. PMID: 26005545, PMCID: PMC4424717. 